\title{
Políticas Científicas actuales en Costa Rica: análisis y potenciales líneas en materia I+D+i
}

Jorge Caldera-Serrano

Universidad de Extremadura, Departamento de Información y Comunicación, Badajoz, España jcalser@unex.es

DOI: https://doi.org/10.26512/rici.v13.n1.28137

Recebido/Recibido/Received: 2019-11-06

Aceitado/Aceptado/Accepted: 2020-01-18

ARTIGOS DE REVISÃO

Resumen: Se analizan y evalúan las políticas en materia de investigación y desarrollo en Costa Rica, llevando a cabo un estudio de caso partiendo de datos objetivos tales como la productividad científica y la inversión en I+D+i llevadas a cabo tanto en el ámbito público como en el privado. Utilizando datos oficiales de plataformas y herramientas para el análisis de la Ciencia, se complementa con el estudio detallado de las políticas actuales que se desarrollan en Costa Rica, analizando para ello el Sistema Nacional de Ciencia e Innovación del Gobierno nacional de manera general y muy especialmente las labores desarrollas por la CONICIT. En último lugar, y como ejercicio de reflexión, se analizan dichas políticas e inversiones públicas, ofreciendo líneas de trabajo y mejoras detectadas en las políticas científicas del país centroamericano.

Palabras claves: Costa Rica. Política científica. Sistema Nacional de Ciencia e Innovación.

\section{Current Scientific Policies in Costa Rica: analysis and potential lines in I+D+i}

Abstract: Research and development policies are analyzed and assessed in Costa Rica, carrying out a case study based on objective data such as scientific productivity and I+D+i investment, carried out both in the public sphere and in the Private. Using official data of platforms and tools for the analysis of science in the country, it is complemented by the detailed study of the current policies that are developed in the country, analyzing for it the National System of science and innovation of the Government of Costa Rica in a general way and especially the work carried out by the CONICIT. In one last place, and as a reflection exercise, these policies and public investments are analyzed, offering potential lines of work and improvement detected in the scientific policies of the Central American country.

Key words: Costa Rica. Scientific policy. National System of Science and Innovation.

Políticas científicas atuais na Costa Rica: análise e linhas de potencial em P + D + i

Resumo: As políticas de pesquisa e desenvolvimento na Costa Rica são analisadas e avaliadas, realizando um estudo de caso com base em dados objetivos, como produtividade científica e investimento em P\&D, realizados tanto em público como em público. no privado. Utilizando dados oficiais de plataformas e ferramentas para a análise da ciência, é complementado por um estudo detalhado das políticas atuais desenvolvidas na Costa Rica, analisando o Sistema Nacional de Ciência e Inovação do governo nacional de uma maneira geral e, principalmente, o trabalho realizado pelo CONICIT. Por fim, e como exercício de reflexão, são analisadas essas políticas e investimentos públicos, oferecendo linhas de trabalho e melhorias detectadas nas políticas científicas do país da América Central.

Palavras chave: Costa Rica. política cientifica. Sistema Nacional de Ciência e Inovação. 


\section{Introducción}

Costa Rica es "pura vida". Esta frase resume claramente la forma de entender la vida de los habitantes del país. Uno de los países con una de las tasas más alta de felicidad, donde el arte del buen vivir no es complicado, donde se vive con humildad frente a la exuberancia medioambiental que nos hace ínfimos y vulnerables. Unas gentes optimistas que disfrutan de la vida, donde se aprecia la vitalidad de lo primario. "Pura vida" que desborda sus tierras, repletas de innumerables bellezas naturales, de paisajes y biodiversidad única e inimaginables. Un país para ir, volver, y no marcharse.

La República de Costa Rica es una nación soberana, presidencialista, ubicada en Centro América. Rodeado por mar y océano (Caribe y Pacífico) y con vecinos panameños y nicaragüenses. Con una población que supera los cinco millones de habitantes es una de las democracias más consolidadas en la siempre inestable América Central, inestabilidad no siempre propia sino incentivadas en ocasiones por elementos externos. País que en el año 1948 abolió la existencia del ejército, lo que queda reflejada en su Constitución de un año más tarde, lo que entendemos como una de las decisiones estratégicas y políticas más destacables de su historia, y que debiera ser copiado por todos para el bien de la Humanidad (WIKIPEDIA, 2018).

Subiendo en todos los índices internacionales, destacamos su índice de desarrollo humano, generado por el Programa de las Naciones Unidas para el Desarrollo (PNUD), índice que pondera la esperanza de vida, tasas de alfabetización y el nivel de calidad vital.

Es destacable igualmente los esfuerzos del país en convertirse en una nación "neutral del carbono", intentando alcanzar este logro para el año 2021, año en que cumplirá sus 200 años del nacimiento como nación independiente (WIKIPEDIA, 2018).

El Happy Planet Index, (2018) en el año 2016, lo encumbró a la primera posición como el estado más ecológico, con una mayor tasa de felicidad, y con políticas medioambientales que garantizan la sostenibilidad de su ecosistema.

Con su capital en San José, donde más de tres millones de personas viven en su zona metropolitana, es el núcleo urbano más importante desde donde se centraliza toda su actividad político-económica, y se regulan sus principales actividades económicas, como son el turismo y la agricultura.

En el ámbito de la Ciencia, la tecnología y la investigación en general, y adelantando algunos datos y conclusiones, Costa Rica ha realizado y realiza grandes esfuerzos por contar con una investigación de calidad en ámbitos estratégicos nacionales, y desarrolla sus investigaciones básicamente en los centros universitarios públicos de la Universidad de Costa Rica y del Instituto Tecnológico de Costa Rica. En las estructuras gubernamentales cuenta con 
dos estamentos claves en esta materia, como son un Ministerio creado con este fin, como es el Ministerio de Ciencia, Tecnología y Telecomunicaciones (MICITT) y la institución que expresamente se encarga del fomento de la investigación en Costa Rica que es el Consejo Nacional para Investigaciones Científicas y Tecnológicas (COSTA RICA, 2018; CONICIT, 2018b).

\section{El Ministerio de Ciencias, Tecnología y Telecomunicaciones (MICITT) y el Consejo Nacional para Investigaciones Científicas y Tecnológicas (CONICIT)}

En el año 1990, en la Ley no 7169 del 26 de junio, se crea la "Ley de Promoción del Desarrollo Científico y Tecnológico, donde se lleva a cabo un gran cambio en las políticas de ciencias y tecnología, ya que en primer lugar se crea el Sistema Nacional de ciencia y Tecnología, cuyo máximo representante sería el Ministerio de ciencia y Tecnología".

El actual Ministerio de Ciencia, Tecnología y Comunicaciones es el encargado de dictar "la política pública de ciencia, tecnología y telecomunicaciones" (Costa Rica, 2018) de tal manera que debe potenciar la optimización y aprovechamiento del conocimiento generado gracias a las acciones políticas y a las sinergias con instituciones públicas y privadas con el fin de mejorar "la competitividad, el bienestar y la prosperidad" (COSTA RICA, 2018), contando con la visión de que el Ministerio deba propulsar un desarrollo integral en el marco del propio país y de su entorno.

Tal y como se señala en las fuentes institucionales, los ejes estratégicos de MICITT son: a) el desarrollo de los recursos humanos en diferentes áreas de la ciencia, la tecnología, la innovación, para que sean el motor de cambio y desarrollo; b) la innovación como competencia clave para la mejora de la calidad de vida de los costarricenses; c) la investigación y el desarrollo, y d) que la mejora de las telecomunicaciones son claves para la vertebración nacional (Costa Rica, 2018).

Los objetivos estratégicos marcados por el Ministerio de Ciencia, Tecnología y Telecomunicaciones están alineados con los ejes estratégicos, por lo que al final desean fortalecer, incentivar y potenciar cada uno de los apartados propuestos anteriormente.

No obstante, sin querer quitarle importancia al Ministerio en el marco del desarrollo científico y tecnológico del país, parece oportuno indicar que, tras la dotación presupuestaria oportuna, las labores de desarrollo de las políticas marcadas por el Ministerio vienen generadas por un organismo autónomo creado para tal fin, como es el CONICIT (CONSEJO, 2018).

El Consejo Nacional para Investigaciones Científicas y Tecnológicas, conocida en el país como CONICIT, es la principal institución en materia de fomento y difusión de la Ciencia y la Investigación, por lo que su análisis pormenorizado es fundamental para determinar las 
principales líneas de trabajo y financiación en materia de ciencia, tecnología, investigación, innovación y desarrollo.

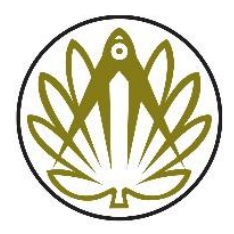

Para el análisis de dicha institución, la principal fuente utilizada ha sido tanto la web como el contacto directo con la institución. EI CONICIT, con la Dra. Giselle Tamayo al frente en la actualidad, debe considerarse como una CONICIT institución autónoma que potencia las principales áreas de investigación en el país tanto desde el ámbito científico como tecnológico. Creada por orden gubernamental cuenta con una historia de 46 años (creada el 22 de agosto de 1972), aunque no será hasta un año más tarde cuando comience su actividad (CONSEJO, 2018b).

No obstante, la institución cuenta con un resurgir en la década de los 80 del siglo XX, cuando cuenta con recursos de la Agencia Internacional para el Desarrollo (AID) y en los años 90 se desarrolla con los Fondos del Banco Interamericano de Desarrollo (BID), comenzando entonces su despegue a través de la financiación de investigación y de la vertebración de un sistema nacional de investigación. Con el cambio de siglo también cambiaron las fuentes de financiación, que han venido a afianzar las políticas de financiación de la investigación por los Fondos PROPYME (Imagen 1).

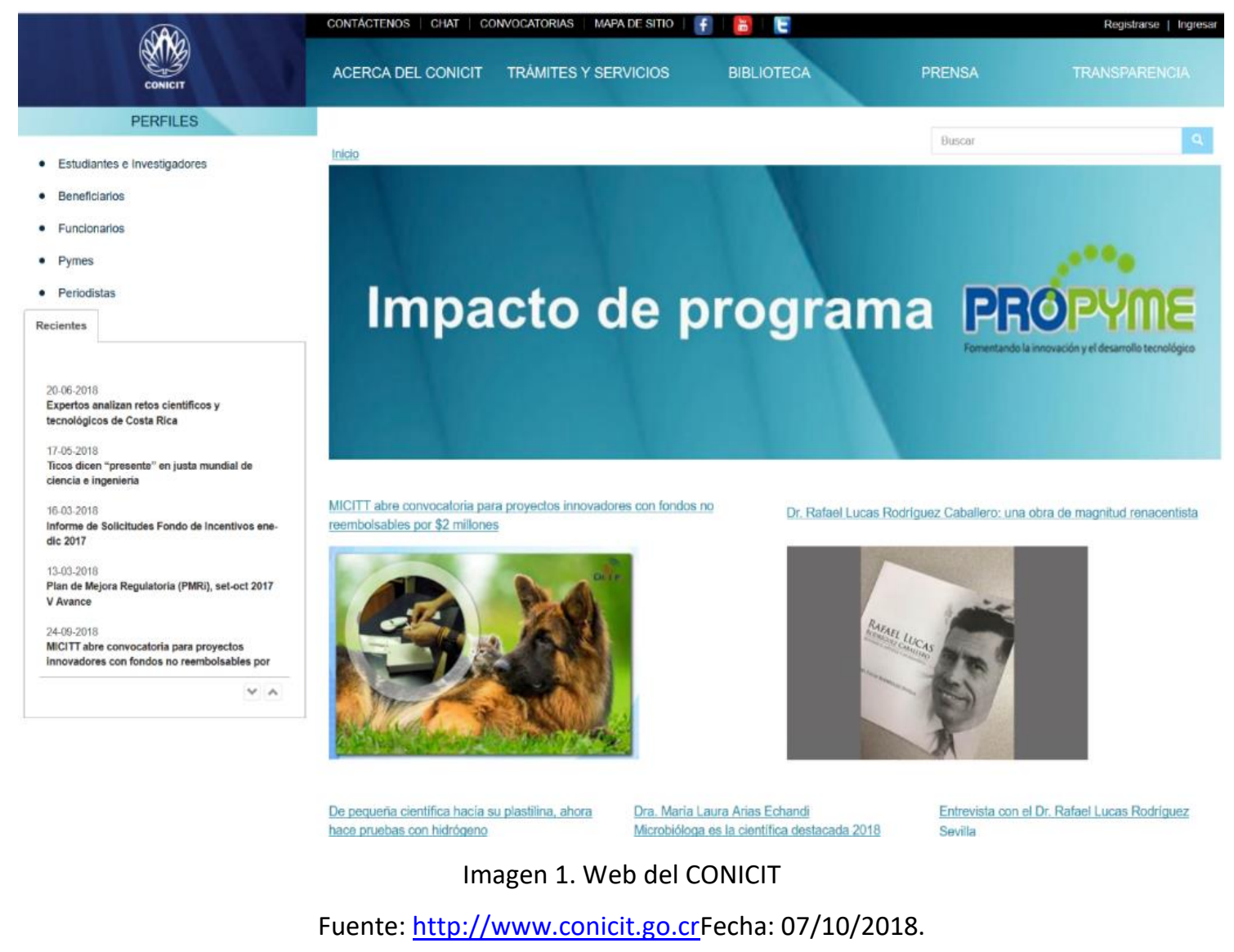


En la propia web del CONICT señala que es la institución de referencia en la "promoción de las capacidades nacionales en ciencia y tecnología, con el apoyo en: la formación de recursos humanos especializados a nivel de posgrado y posdoctorado, el financiamiento de proyectos de investigación básica y aplicada, de desarrollo experimental, de desarrollo tecnológico, infraestructura física y de equipo a centros de excelencia científica, capacitaciones a corto plazo de investigadores, participación en eventos nacionales e internacionales y el manejo de inventarios nacionales de recursos humanos, centros de investigación y proyectos de investigación" (CONSEJO, 2018b). Sin lugar a dudas, este párrafo que describe la institución marca su potencialidad y su línea de actuación.

Igualmente es destacable señalar los principales retos estratégicos de la institución, los cuales se resumen en los siguientes cinco puntos: "Consolidar una gestión estratégica orientada a resultados para mejorar la calidad de vida de la sociedad costarricense. Construir una cultura de dedicación a la comunidad objetivo que oriente las relaciones de todos los niveles de la organización con las partes interesadas. Fortalecer el compromiso y aprovechar las capacidades y potencialidades del equipo humano CONICIT para el logro de los objetivos institucionales. Desarrollar y consolidar un modelo de sucesión sustitutivo y de contingencia en la institución. Diseñar y desarrollar el enfoque de trabajo por procesos" (CONSEJO, 2018b).

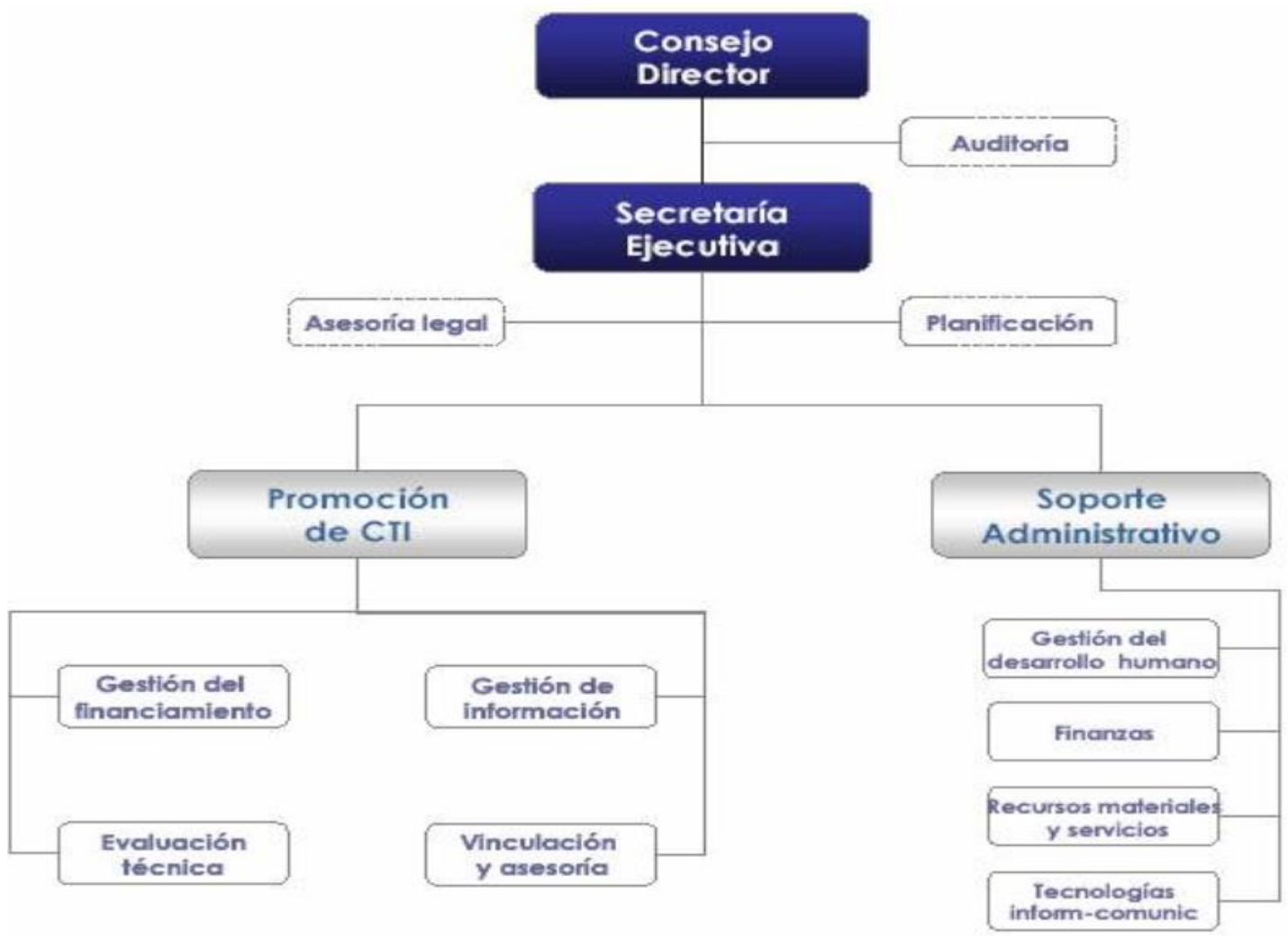

Imagen 2. Estructura organizacional CONICIT

Fuente: http://www.conicit.go.cr/acerca de/estructura/index.aspxFecha: 07/10/2018 
En lo que respecta a la estructura de la organización, señalar la existencia de un Consejo Director, conformado por cinco miembros, que es el órgano máximo y autónomo para la toma de decisiones. Mientras que será desde la Secretaría Ejecutiva desde la que se lleva a cabo la Promoción de la Ciencia. La sección denominada Soporte Administrativo es la encargada del necesario control y gestión de todos los procedimientos. Previo a ello, la Secretaría Ejecutiva controla tanto la Asesoría Legal como el Soporte Administrativo.

EI CONICIT entrega diferentes premios para potenciar la labor investigadora, así como su difusión. Destacamos el Premio TWAS/CONICIT para científicos jóvenes menores de 40 años (regularidad anual, en las áreas de la biología, química, física y matemáticas), el Premio a la Empresa Editorial que mayor difusión haga de la Ciencia y la Tecnología, el Premio a la Innovación y Tecnología, especialmente para PYMES y en colaboración con la Cámara de Industrias de Costa Rica, y el Premio CONICIT para científicos y tecnólogos jóvenes.

EI CONICIT cuenta con cuatro programas para el desarrollo de sus políticas:

- Programa de reinserción de investigadores, cuya última convocatoria se cerró en julio de 2017, con un total de 6 proyectos financiados. Convocatoria centrada hacia las universidades públicas y privadas como otras instituciones de investigación del país, con la intención de integrar a nuevos doctores y doctorandos en los proyectos de investigación en áreas científico tecnológicas, con el fin de generar producción de "alto impacto" (concepto muy ambiguo presente en muchas legislaciones de todo el mundo) (CONSEJO, 2018 b).

- Programa de Residencia Temporal en Alemania para empresarios costarricenses. Programa muy concreto para asistir a un seminario en Baviera (Alemania). Aunque este proyecto de formación no debe entenderse como un programa destinado a la investigación, parece oportuno señalar el esfuerzo, en colaboración con otras instituciones, por ayudar e integrar a los empresarios costarricenses en nuevos modelos productivos. No obstante, no estimamos muy oportuno y correcto que sea entendido como un programa en el marco de la CONICIT sino como una acción temporal para un determinado evento.

- Programa Fondo PROPYME. Tal y como señala la propia web del CONICIT, el programa está orientado a "financiar las acciones y actividades dirigidas a promover y mejorar la capacidad de gestión y competitivas de las pequeñas y medianas empresas costarricenses, mediante el desarrollo tecnológico e innovación como instrumento para contribuir al desarrollo económico y social de las diversas regiones del país" 
(CONSEJO, 2018 b). Por lo tanto, centrado en las PYMES, cuenta con diferentes convocatorias a lo largo del año, prácticamente una mensual.

- El Programa de Fondos de Incentivos ofrece diferentes convocatorias a lo largo del año para presentar solicitudes de financiación para proyectos de investigación en "virtud del cumplimiento de los objetivos y políticas científicas y tecnológicas planteadas en los instrumentos de planificación institucional" (CONSEJO, 2018b). Extrapolando el concepto, podríamos denominarlo como el Plan Nacional de Investigación, en el cual se facilitan recursos a los grupos de investigación para desarrollar diferentes labores, orientadas a las mejoras de los ejes estratégicos de Costa Rica.

\section{Estado de la producción científica de Costa Rica}

Son cada vez más los estudios que analizan y miden la Ciencia desde diferentes prismas. No obstante, desde las Ciencias de la Información es cada vez mayor la literatura científica centrada en el análisis métrico de la literatura científica, con el fin de servir de herramienta y arma organizativa para determinar políticas en materias retributivas de incentivos, así como para la ayuda en la toma de decisiones para determinar la financiación pública de proyectos de investigación. El estudio de las fuentes de procedencia de los trabajos está provocando innumerables problemas, siendo actualmente los estudios métricos un ámbito de trabajo muy interesante especialmente por la búsqueda de nuevos parámetros para la medición de la Ciencia.

Aunque creemos firmemente que el factor de impacto de las fuentes de publicación no es ni mucho menos la mejor fórmula para la determinación de la calidad de la producción científica, como bien es sabido sigue siendo el patrón fundamental y prácticamente único para evaluar las disciplinas científicas y los individuos que en ella cohabitan.

Una vez determinada nuestra postura sobre dichos parámetros y herramientas, y centrándonos propiamente en nuestro trabajo, vamos a utilizar para cuantificar y analizar el estado actual de la producción científica en Costa Rica partiendo de las posibilidades existentes, de herramientas que en algunos casos, no son completas ni universales, que discriminan por el idioma, por áreas e incluso por empresas editoriales, pero que son a día de hoy de las pocas fórmulas que tenemos para analizar dicha producción. Por lo tanto, tanto los datos como la posición en los diferentes rankings no siempre son absolutos, y en cierta manera los resultados están supeditados a los hábitos investigadores y de difusión.

Ciertamente no toda investigación acaba en publicación, aunque en el sistema y proceso actual la difusión de los resultados científicos se nos plantea como imprescindible, especialmente en ámbitos universitarios, de instituciones de investigación y en todo el 
entramado de proyectos financiados con ayudas públicas, no obstante en muchas ocasiones quedan fuera del sistema de difusión de la investigación aquellas realizadas por instituciones privadas ya que, por su misión y funcionalidad, no suelen compartir sus resultados hasta haber sido completados, y normalmente, en forma de patente que blindan los derechos de autor y de explotación.

$Y$ aunque como ha podido establecerse anteriormente, somos abiertamente críticos con el modelo actual que utiliza el factor de impacto de las revistas como modelo y eje fundamental, es imprescindible utilizarlas. En nuestro caso para conocer el estado de publicación en Costa Rica hemos utilizado la herramienta del grupo de investigación Scimago (2018), realizada para Scopus. Con esta herramienta se identifica la posición de Costa Rica en su entorno (América Central) y a nivel mundial, destacando aquellas áreas en la que la producción presente en Scopus es mayor.

Además de los datos comparativos y absolutos extraídos de la herramienta generada por el grupo Scimago, vamos a plantear otras cuestiones tales como inversión y número de investigadores dedicados a la investigación siguiendo para ello una aplicación solvente y contrastada como Index Mundi (2018), la cual recoge la información de diferentes fuentes en muchos casos públicas y siempre de contrastada reputación. Igualmente utilizaremos información extraída de las herramientas del Banco Mundial (2018).

Diferentes autores, entre los que destacamos a Piedra y Martínez (2007), señalan que las investigaciones se ponen a disposición de la comunidad científica básicamente por prestigio ante sus colegas y por el desarrollo de la carrera profesional. Por lo tanto, existe una doble función: de posicionamiento en la disciplina y por la búsqueda de incentivos económicos personales, institucionales y de financiación de futuras investigaciones. Es de sobra conocido el "Efecto Mateo", por el cual a un investigador citado se le suele citar más por el simple hecho de convertirse en referente, pues igualmente pasa con la financiación para proyectos de investigación: aquellos directores de proyectos que han dirigido grupos capacitados y con resultados brillantes estarán en disposición de obtener mejores resultados en próximas convocatorias competitivas de investigación. Por lo tanto, se publica para poder seguir investigando y así contar con la materia prima para obtener nuevos resultados que nos otorguen nuevas publicaciones. Una espiral en constante movimiento.

En el marco de la producción científica, lo habitual es la difusión en forma de artículo científico en revista, lo que ha desbancado prácticamente en todas las disciplinas a monografías y actas de congreso, lo que supone una merma de representatividad en herramientas como SCOPUS y WoS para las áreas de las Ciencias Sociales y Humanas que aún siguen usando el libro y las tradicionales actas de congreso como modelo de difusión. 
En la imagen 3 puede apreciarse un cuadro general con la producción de Costa Rica, comparándola con los países cercanos. En este caso aparecen todos los países de América Latina. Analizando la producción recogida desde el año 1996 hasta 2017 (ambos años incluidos) la producción total de trabajos presentes en las herramientas con autores o instituciones de Costa Rica es un total de 11.445, ubicando al país en el puesto 12 en la región, y en primer lugar de los países de América Central, con una clara superioridad frente a Panamá que se encuentra en el puesto 13 pero con un número importante de documentos de desventaja (6.349 documentos registrados).

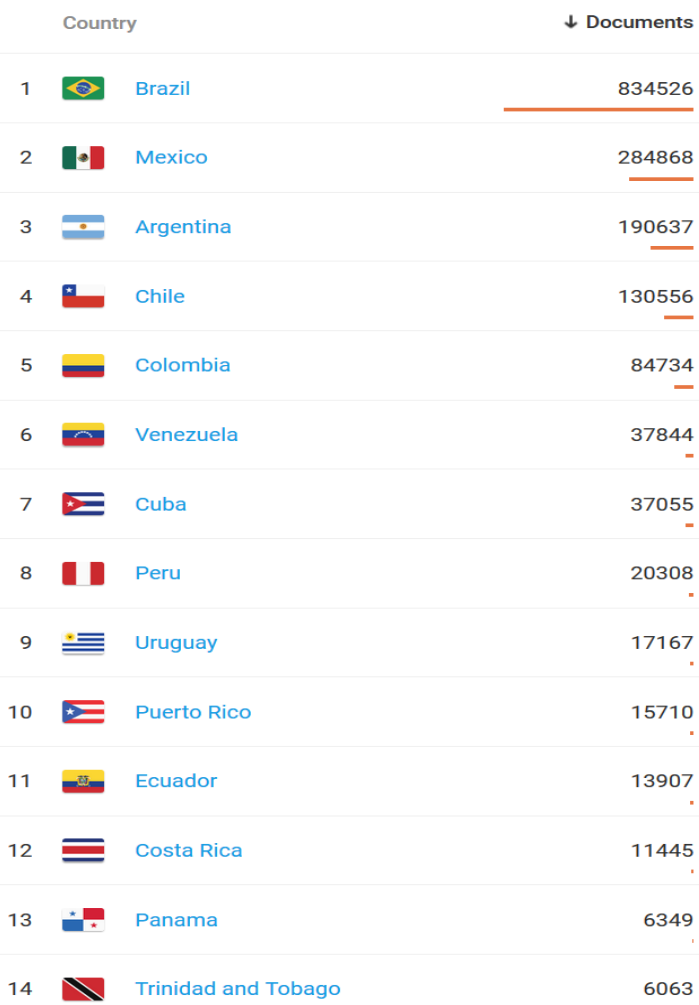

Imagen 3: Producción científica total en Scopus. Filtro: América Latina Fuente: https://www.scimagojr.com/countryrank.php?region=Latin\%20America Fecha: 28 de septiembre de 2018

El dato del número total en la producción científica presente en Scopus de Costa Rica en el contexto latinoamericano daría como resultado que el peso en la producción regional es de un escaso $0.66 \%$, nada comparable con los primeros puestos donde Brasil, con 834.526 documentos supone un porcentaje del 48,35 lo que la coloca de forma clara como la principal potencia científica en la región; seguida de México, cuya producción con 284.868 registro supone un $16,5 \%$ de la producción de la región; y destacamos dentro de los tres primeros puestos la producción de Argentina con un total de 190.637 registros que le otorga un 11,04\%. Pero esta comparativa no es realmente justa, aunque real. Los parámetros de Costa Rica no 
son equiparables con los tres grandes gigantes de Latino América, y sus datos son difícilmente comparables.

La tabla 1 siguiente, elaborado a partir de los datos obtenidos en Scopus, nos facilitará una mejor visión no de la presencia de Costa Rica en Centro América.

Tabla 1: Producción científica total en Scopus. América Central

\begin{tabular}{|l|c|c|c|}
\hline \multicolumn{1}{|c|}{ País } & Número de registros & Porcentaje (\%) & Posición en Centro América \\
\hline Belice & 405 & 1,59 & $\mathbf{7}$ \\
\hline Costa Rica & $\mathbf{1 1 . 4 4 5}$ & $\mathbf{4 5 , 0 0}$ & $\mathbf{1}$ \\
\hline Guatemala & 2.910 & 11,44 & 6 \\
\hline Honduras & 1.252 & 4,92 & 4 \\
\hline Nicaragua & 1.597 & 6,28 & 2 \\
\hline Panamá & 6.349 & 24,96 & 5 \\
\hline El Salvador & 1.470 & 5,78 & 3 \\
\hline
\end{tabular}

Datoshttps://www.scimagojr.com/countryrank.php?region=Latin\%20America Fecha: 28 de septiembre de 2018.

A la vista de los datos, Costa Rica se nos muestra como la gran generadora de producción científica en América Central, sumando junto a Panamá prácticamente el 70\% de la producción regional. Los datos aportados por otros países son bastante bajos ya que Guatemala supera escasamente el 10\% mientras que el resto (Nicaragua y El Salvador superan el $5 \%$ y el $6 \%$, respectivamente). Datos especialmente pobres son los aportados por Belice, los cuales tienen que ver con las características propias del país.

Evidentemente, y utilizando lenguaje futbolístico, Costa Rica es el primer clasificado de la segunda división, la pregunta que hemos de hacernos es si realmente la potencialidad del personal investigador y de la financiación institucional debería tener mejor resultados de los previstos o deberíamos estar orgullosos de dicha producción. Vamos a analizar otros elementos que nos podrán ayudar a contestar a dicho dilema.

Antes, no contante, nos gustaría resaltar la producción presente en Scopus desde el año 2000 al 2017, para evaluar el cambio en la posición en el ranking de países de América Latina, así como si existe un aumento significativo a lo largo del tiempo.

Tabla 2: Producción científica de Costa Rica en Scopus: 2000-2017

\begin{tabular}{|c|c|c|c|}
\hline Año & Registros & Porcentaje (\%) & Posición América Latina \\
\hline 2000 & 228 & 2,18 & 11 \\
\hline 2001 & 283 & 2,76 & 10 \\
\hline 2002 & 301 & 2,88 & 10 \\
\hline 2003 & 308 & 2,95 & 11 \\
\hline 2004 & 412 & 3,95 & 11 \\
\hline 2005 & 445 & 4,27 & 11 \\
\hline 2006 & 459 & 4,40 & 11 \\
\hline 2007 & 515 & 4,94 & 11 \\
\hline
\end{tabular}




\begin{tabular}{|l|l|l|l|}
\hline 2008 & 524 & 5,03 & 11 \\
\hline 2009 & 563 & 5,40 & 11 \\
\hline 2010 & 597 & 5,73 & 11 \\
\hline 2011 & 640 & 6,14 & 11 \\
\hline 2012 & 696 & 6,68 & 11 \\
\hline 2013 & 712 & 6,83 & 11 \\
\hline 2014 & 889 & 8,53 & 11 \\
\hline 2015 & 859 & 8,24 & 11 \\
\hline 2016 & 956 & 9,17 & 11 \\
\hline 2017 & 1031 & 9,89 & \\
\hline
\end{tabular}

Datos: https://www.scimagojr.com/countryrank.php?region=Latin\%20America. Elaboración propia.

Fecha: 28 de septiembre de 2018

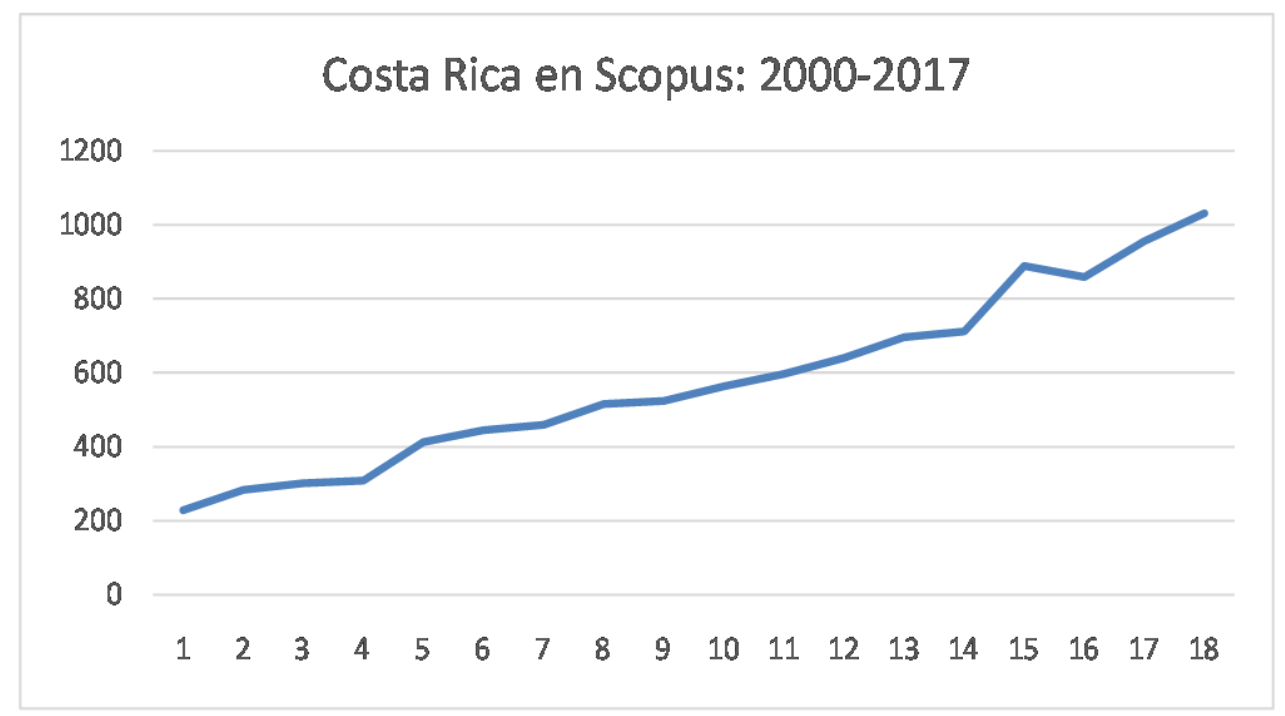

Gráfico 1: Producción de Costa Rica en Scopus 2000-2017

Datos: https://www.scimagojr.com/countryrank.php?region=Latin\%20America. Elaboración propia.

Fecha: 28 de septiembre de 2018

Como puede apreciarse a la vista de la tabla y gráfico anteriores, el aumento en la producción científica de los investigadores costarricenses en Scopus son constantes, pero a priori no significativa. Pueden observar como la posición en el ranking latinoamericano de producción es prácticamente constante, siempre está presente en el puesto número 11, salvo en años puntuales con bajada a la posición 12 y subida al 10, aunque eso ocurrió en el año 2001 y 2002. Por lo tanto, podríamos determinar que dicha tendencia de subida, sólo interrumpida en el año 2014 por no muchos registros -por lo que igualmente no es significativa- puede implicar varias posibilidades: la primera de ellas es que no haya habido incentivos suficientes, o al menos novedosos, en el ámbito de la investigación y desarrollo de proyectos que pudieran dar como resultados mayor producción. Por lo tanto, no parece que haya habido políticas de investigación arriesgadas o supone que todos los países de América 
Latina han tenido las mismas iniciativas, de tal manera que todos hayan subido por igual (lo que no parece aparentemente muy probable). Una segunda opción, bastante plausible, es que el aumento constante en el número de publicaciones presente en Scopus haya hecho posible el aumento en el número de trabajos de autores costarricenses.

No obstante, existen elementos que nos pueden ayudar a determinar este aumento en los números de Costa Rica, como pudiera ser el aumento en la inversión en I+D, mejores y mayores recursos para el aumento de los recursos humanos, internalización de la investigación y de las relaciones de coautorías, etc.

Un factor determinante en el aumento de la producción y en la mejora de la presencia en esta y en otras bases de datos es la inclusión de revistas propias que tuvieran una mayor presencia de producción nacional. A mayor número de revistas de un país, mayor producción de los investigadores de este país. Lo que parece normal, habida cuenta que los mayores lectores, consumidores e investigadores de una revista será los investigadores de dicho entorno.

Costa Rica únicamente cuenta con tres revistas presente en la base de datos Scopus, lo que nos da a entender claramente que existe una producción importante de investigadores costarricenses en revistas extranjeras, ya que no puede mantenerse los números descritos con únicamente tres revistas.

Parece interesante y oportuno mencionar estas tres revistas, para mostrar un camino a seguir para la mejora de la visibilidad y del aumento de la producción y relación internacional.

Lankesteriana, revista publicada por la Universidad de Costa Rica en la categoría de "Agricultural and biological Science - Plan Science", presente desde el año 2005 (con su desaparición en Scopus desde 2011 a 2013) y que desde su vuelta ha subido en la visibilidad hasta encontrarse desde el 2015 en el segundo cuartil de la disciplina.

La segunda revista es la publicada por la Universidad de Costa Rica bajo el título Revista de Biología Tropical. Presente en la disciplina de "Agricultural and biológica Sciences" (al igual que la anterior) en la subcategoría "Agricultural and Biological Science (miscellaneous). Revista con gran visibilidad internacional desde el año 1999, ocupando prácticamente todos los años una posición en el segundo cuartil.

Y frente a las dos revistas anteriores, con similitudes de disciplinas evidentes, nos encontramos con la Revista de historia, editada por la Escuela de Historia de la Universidad Nacional, que independientemente de que su título pudiera llevarnos a confusión está inscrita en la categoría de "Medicine (miscellaneous)". Revista bien posicionada en el año 2004, al estar el cuartil tres, despareció ese mismo año de Scopus, siendo indexada únicamente desde el año 2000. 
Por lo tanto, es importante determinar que actualmente son solo dos revistas las presente en esta base de datos, en temáticas estratégicas en la investigación en un país tan marcado por su geografía y condicionantes medioambientales como es Costa Rica.

No obstante, y poniendo a Costa Rica en el contexto mundial, el número de trabajos presente en Scopus desde el año 1996 posicionan al país en el puesto 93 de un total de 239 países analizados en la herramienta de Scimago, con dos únicas revistas de un total de 34.171.

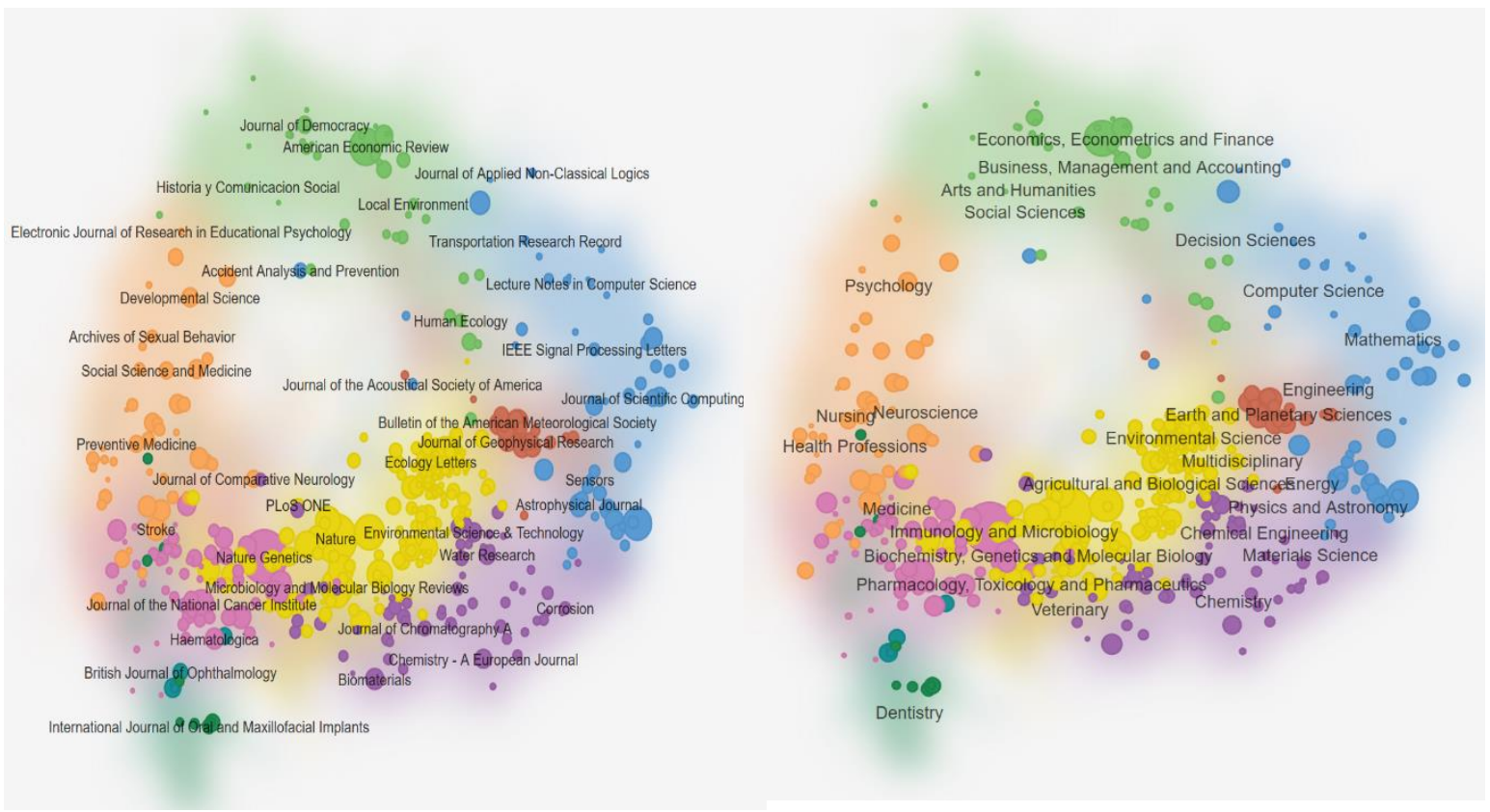

Imagen 4: Revistas y áreas en las que publican los autores con nacionalidad costarricense

Fuente: http://www.scimagojr.com/shapeofscience Fecha: 07/10/2018

Se puede apreciar en la imagen 4 dos cuestiones importantes. La primera de ellas nos informa en qué revistas publican los autores analizados, mientras que en la segunda nos especifica el área de producción. Independientemente de las revistas mencionadas, lo que sirve para identificar publicaciones concretar, es interesante destacar la internacionalización de los resultados de los investigadores de Costa Rica, lo que supone estar presente como autor único o en coautoría en revistas visibles en todo el Planeta. Y todo ello siendo consciente de los problemas de estas bases de datos en la inclusión de revistas en habla no anglosajona, por lo que obliga a estos investigadores a publicar y a relacionarse normalmente en inglés y con autores angloparlantes. Esto a priori no debiera ser problema si no fuese porque al final generamos una producción que no siempre es accesible por la ciudadanía (por el idioma) ni por la comunidad científica del país, la cual de pagar cuantiosos aranceles por conseguir dichos documentos. 
Entendemos más relevante la segunda imagen, en la cual se analiza en qué áreas se llevan a cabo la difusión de los resultados presente en la base de datos Scopus y, seguramente, en qué disciplinas se aporta mayores recursos económicos para investigación (esta correlación suele ser real en la mayor parte de los casos).

Observen como la mayor producción está concentrada en las áreas relacionadas temáticamente en color amarillo en el gráfico. Agricultural and Biological, Enviromental Science, en definitiva, todo lo relacionado con las Ciencias de la Tierra tienen un lugar destacado en la producción científica. Valga recordar que Costa Rica cuenta con el mayor, y seguramente mejor, laboratorio natural para desarrollar investigaciones relacionadas con la materia indicada, siendo sin lugar a dudas, un país especial para el desarrollo de estudios medioambientales. También destacado, y muy relacionado con la potencialidad natural del país, se observa una importante producción de farmacología, toxicología, medicina, bioquímica, mezclado y enlazado con las Ciencias de la Vida y estudio del Planeta.

El lector puede sacar múltiples y diferentes lecturas de las imágenes anteriores, pero queremos destacar no sólo los puntos fuertes, en qué se publica más en Costa Rica, sino también centrarnos en las carencias evidentes en disciplinas que entendemos igualmente importantes. Parece que la investigación está centrada en la aplicación práctica, dejando de lado en general las Artes, las Humanidades, y muy especialmente las Ciencias Sociales en general. Un grave error estratégico para un país si se elimina del interés disciplinas humanísticas, sin lugar a dudas menos pujantes económicamente pero igualmente fundamental para el desarrollo de un país.

Entendemos interesante cambiar de herramienta de análisis con el fin de analizar otros parámetros que consideramos fundamentales para analizar las políticas en materia de investigación y desarrollo, lo cual no siempre se traduce en publicaciones ya que no toda investigación se convierte en publicación, ya que pueden dar como resultados informes internos, patentes, investigaciones básicas muy valiosas para la comunidad a la que sirve pero con poca relevancia a nivel internacional, o investigaciones locales que siendo valiosas están fuera de las fuentes presentes en las herramientas internacionales.

La web Indexmundi no genera información, sino que vuelca de diferentes instituciones oficiales aquellas que consideran que puede ser útil para su público objeto. Desde este portal se puede acceder a información y datos de las principales agencias internacionales, por lo que consideramos los resultados aportados como información solvente, contrastada y destacable.

En primer lugar, analizamos el parámetro de inversión en I+D. Que dato más relevante desde la promoción pública de la investigación que el montante económico respecto al Producto Interior Bruto del país dedica a la investigación. Tal y como señala la web 
Indexmundi los gatos en investigación y desarrollo son aquellos gastos corrientes y también de capital, tanto público como privado, realizado para el incremento del conocimiento sobre diferentes aspectos tales como la cultural, la sociedad, las nuevas aplicaciones, etc., pudiendo ser investigación básica, aplicada como desarrollo experimental.

Los datos obtenidos del Instituto de Estadística de la Organización de las Naciones Unidas para la Educación, la Ciencia y la Cultura (UNESCO, 2018) le otorga a Costa Rica la posición 63 de 129 países analizados (otorga el primer puesto con un 4.04 del PIB en inversión en investigación y desarrollo a la República de Corea, mientras que el valor más bajo es para Lesotho, con una inversión del 0.01). El PIB de Costa Rica en materia de Investigación está en el 0.48 por ciento, que tal y como se observa en la imagen posterior es la mayor de toda la región.

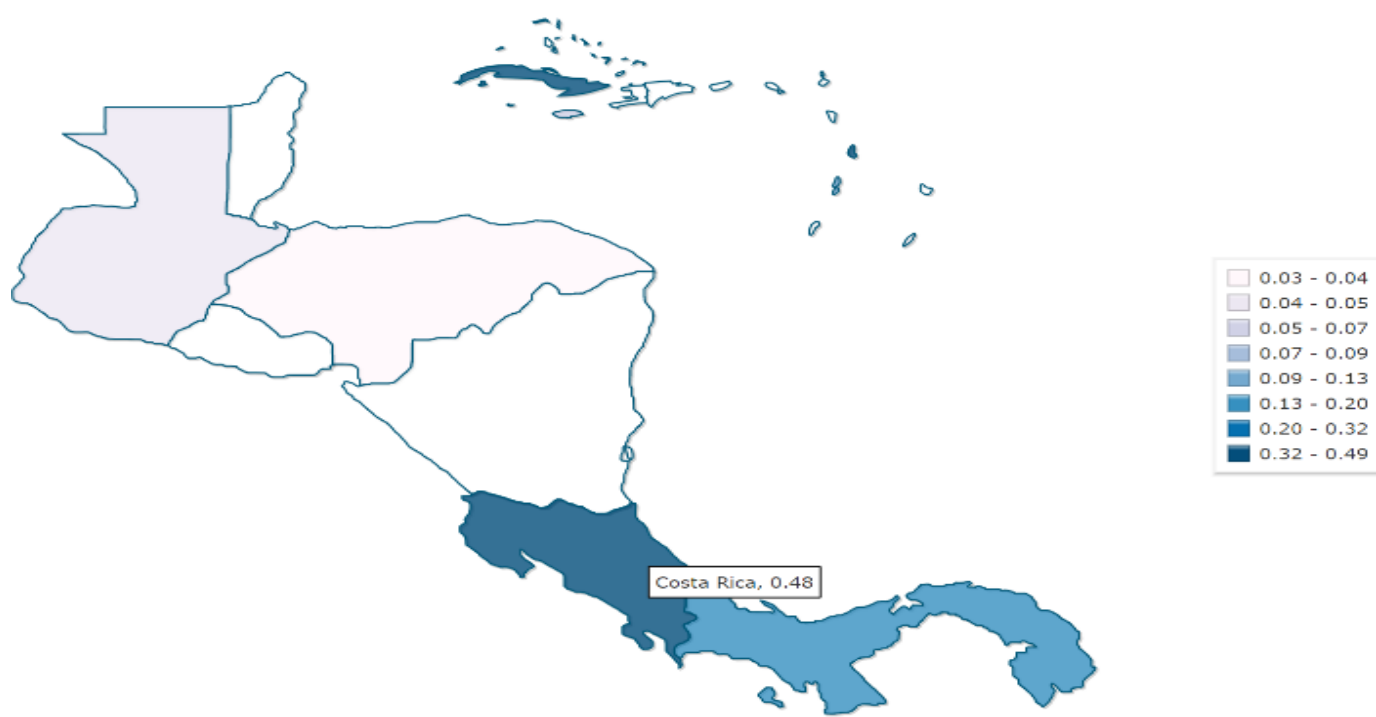

Imagen 5: Inversión en I+D (\% PIB). América Latina y El Caribe.

Fuente: https://www.indexmundi.com/es/datos/indicadores/GB.XPD.RSDV.GD.ZS/map/central-america Fecha: 07/10/2018

No obstante, el Banco Mundial, utilizando la misma fuente (UNESCO) otorga datos similares y más recientes, de tal manera que señala un gasto en PIB de Costa Rica del 0,577 en el año 2014. Lo que la gráfica nos señala, y es sin lugar a dudas importante, es la tendencia al alza en la inversión en este apartado, aunque entendemos que aún está muy lejos de la media mundial que en el año 2014 estaba en el 2,153, y subiendo en el año 2015 al 2,227 (último año del cual se tienen datos). Independientemente de los diferentes estudios que colocan un umbral u otro para determinar el valor adecuado, la inversión de Costa Rica está muy alejado sin lugar a dudas de la media, factor al que se debería intentar alcanzar en el marco de políticas estratégicas nacionales. 


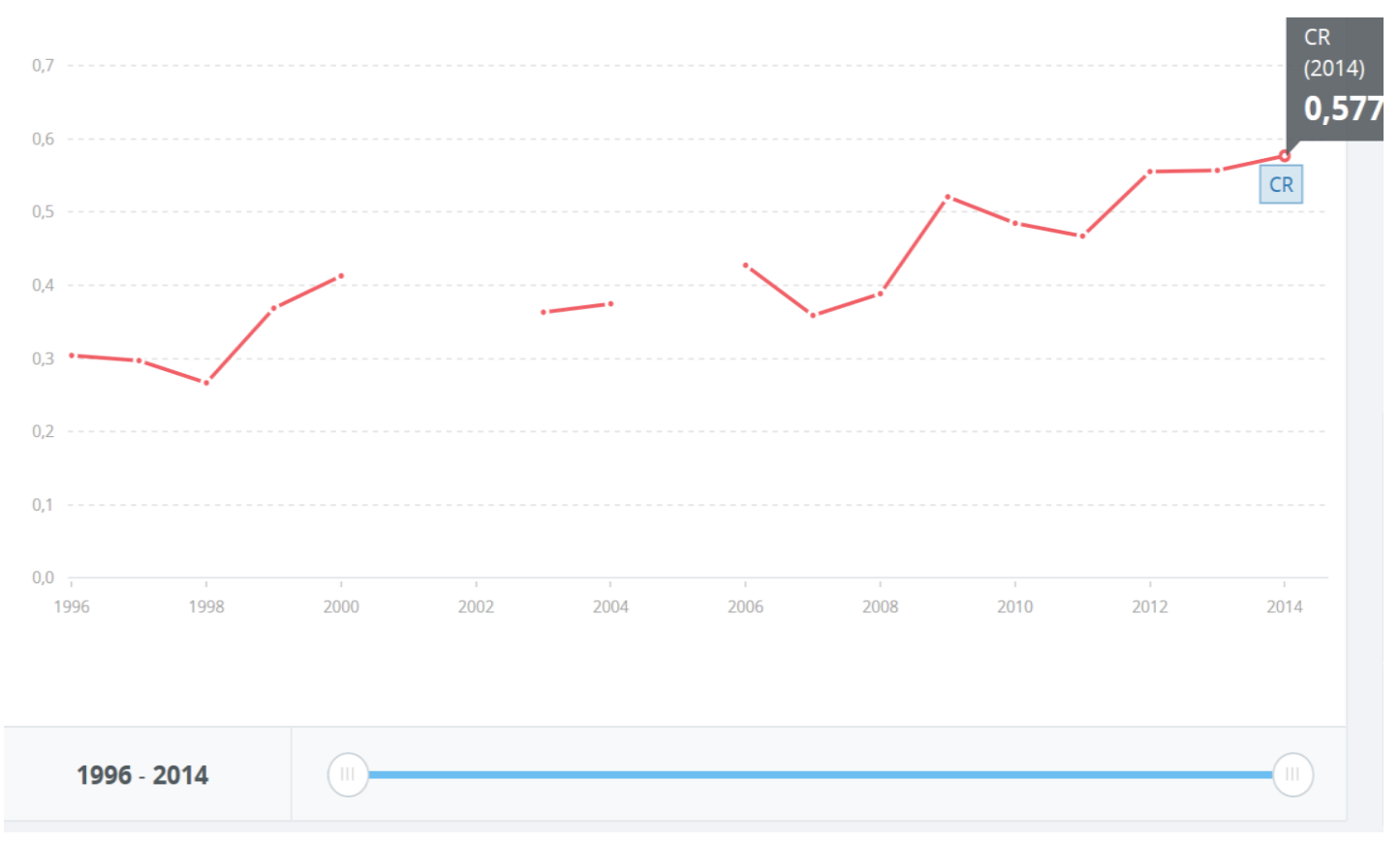

Gráfico 2. Banco Mundial. Gasto en Inversión y Desarrollo (\% del PIB). Datos de la UNESCO

https://datos.bancomundial.org/indicador/GB.XPD.RSDV.GD.ZS?locations=CR Fecha: 07/10/2018.

Otro de los parámetros que pueden tenerse presente y que es muy importante (relacionado con el anterior) es el número de investigadores existentes por cada millón de habitantes. En definitiva, conocer el esfuerzo inversor atendiendo al número de investigadores lo que también nos dará pautas sobre la productividad de los mismos (poniéndolos en relación con la media mundial, y comparándolo con otros países del entorno).

Como puede observarse en la imagen posterior, el número de investigadores ha subido de forma clara en los últimos años en Costa Rica, pasando de los algo más de cien investigadores por cada millón de habitantes en el año 2003, a pasar a los prácticamente 1.300 investigadores en el año 2001. Dicho cambio debe ser analizado atendiendo a políticas de inversión o aumento en el número de instituciones que se dedican a la investigación, ya que desde el año 2008 se observa una tendencia al alza absolutamente plausible. Tanto es así, que para el año 2010 la UNESCO otorgaba una media en el número de investigadores de 1,277, por lo que podemos señalar que de estar muy por debajo de la media en el número de investigadores, actualmente se encuentra en la media (dato aportado por la web del Banco Mundial con datos de la UNESCO). 


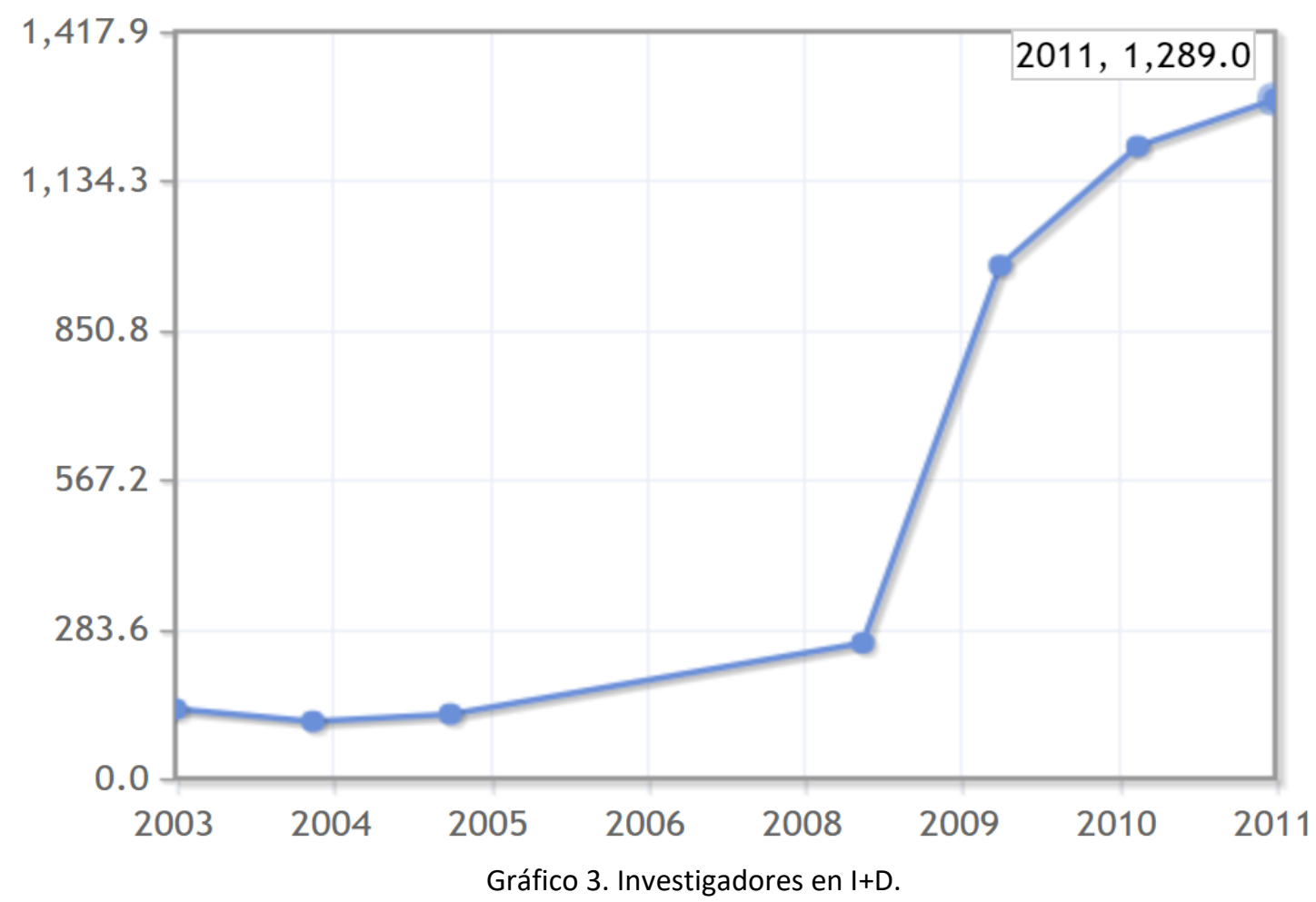

https://www.indexmundi.com/es/datos/costa-rica/investigación-y-desarrollo\#SP.POP.SCIE.RD.P6 Fecha: 07/10/2018.

Aunque los números son muy importantes, ya que se observa el incremente rápido y paulatino en los últimos años, la situación de partida era muy escasa. Queremos aportar un dato ofrecido igualmente por el Banco Mundial quién para los países de la OCDE le otorga un dato medio de investigadores por cada millón de habitantes muy cercano a los 4000 investigadores $(3.960,988)$, lo que sí nos ofrece una distancia importante frente a países desarrollados que, sin duda, son países mucho más potentes en el ámbito de la investigación y el desarrollo. No obstante, tengamos en cuenta que no todos los países de la OCDE van al mismo ritmo, de tal manera que Noruega cuenta en el año 2014 con 5.679 y la República de Corea con 6899, mientras que otros países no llegan a los 500 (Chile, 427).

No obstante, para entender igualmente estos números hemos de ser conocedores que en este campo también se incluye los estudiantes de doctorado, lo cual sigue siendo una tarea pendiente en muchos países latinoamericanos, al no contar con estructurar universitarias que alberguen este tipo de estudios.

Se ha intentado llevar a cabo un diagnóstico por un lado de los esfuerzos normativos y regulatorios del Gobierno de Costa Rica para después poder analizar, con números, la presencia y posicionamiento del país a nivel general utilizando herramientas de análisis cuantitativo, con el fin de ver si existe una correlación entre el esfuerzo económico en políticas científicas, y la presencia internacional de la investigación costarricense. 


\section{Discusión y líneas de actuación}

Como se puede intuir tras la lectura del trabajo, el CONICIT (2018) es realmente quién se encarga, por mandato del Ministerio de Ciencia, Tecnología y Telecomunicaciones, de la puesta en marcha de los planes nacionales y de la inversión en materia de ciencia, investigación e innovación. Por lo tanto, el análisis de este organismo es quien nos aporta las pautas para determinar el grado de apuesta gubernamental de Costa Rica por la inversión en Investigación y en Ciencia.

Entendemos, a priori, que son escasos los Programas existentes, pero sí que existe un esfuerzo permanente en los Programas semiabiertos de ayuda a las PYMES y de realización de proyectos de investigación que tratan sobre los ejes estratégicos del país, o al menos, de la ciencia en Costa Rica. Nos referimos que son programas semiabiertos desde el momento en que prácticamente existen convocatorias mensuales en alguno de los casos, lo que se supone que la CONICIT de forma constante está desarrollando labores de gestión, evaluación y valoración y financiación. No es normal esta labor y de esta manera, normalmente otros países tanto del entorno como europeos, los planes nacionales de investigación se vertebran en momentos muy puntuales con una única convocatoria al año, de gran cuantía, pero única.

Al ser ambos programas de marcado carácter competitivo, la búsqueda de la excelencia y la calidad es uno de los elementos fundamentales, además de centrarse en temáticas muy precisar. Esto, como ya se ha podido corroborar en algunos apartados del trabajo, hace poco viable el desarrollo de disciplinas humanas, artísticas y aquellas relacionadas con las ciencias sociales, incluso ciertos ámbitos tecnológicos están fueran de las marcadas pautas temáticas que se potencian y se financian desde el Gobierno, vía CONICIT. Esto es una debilidad clara, que viene marcada por la cuantía de la financiación total con la que cuenta la CONICIT. Estamos convencidos de que, si la financiación fuese mayor, seguramente, se diversificaría la temática. No obstante, sí que entendemos que se deben realizar mayores esfuerzos de inversión en materia de investigación y desarrollo por parte del Estado, para intentar equipararse a otros países desarrollados.

Su posición privilegiada en la región (primera posición en los rankings analizados de América Central) nos debe ofertar la pauta de lo mucho que debe mejorar la región y no únicamente que Costa Rica se encuentre en una buena posición. La situación es mejorable. Se debería diversificar la oferta y las posibilidades de investigación, implementando potenciales líneas y vías de trabajo, invirtiendo e incentivando la inversión privada, y mejorando el Sistema Nacional de Ciencia, mejorando e incentivando a los centros de investigación, especialmente a 
las universidades que parece son el mayor y mejor motor del desarrollo de la investigación en el país.

No aparece cuestiones que aparentemente debieran tratarse de forma clara y contundente en investigación y desarrollo, por lo que entendemos que las posibilidades de mejoras son muchas, sin quitar importancia al gran esfuerzo del gobierno de Costa Rica, a la buena gestión del CONICIT y de la importante visibilidad de los trabajos de los investigadores del país. Algunos elementos aportados son contradictorios, como contar con un número de investigadores en la media mundial, y sin embargo contar con una baja inversión en relación al PIB.

Se van a aportar una serie de ideas, líneas de trabajo y pautas potenciales para la mejora o implementación de políticas de ciencia, investigación, desarrollo y/o innovación. Evidentemente son recomendaciones a la vista de la realidad de otros países de América Latina y la tendencia en inversión en Ciencia y Tecnología en otras zonas del Planeta, especialmente Europa. Entendemos que es complicado y económicamente poco viable el desarrollo de una política que cubriese los diferentes elementos que se sugieren. En un trabajo previo en el cual se analiza las políticas de la SENACYT llevadas a cabo de Panamá (CalderaSerrano, imprenta), sugiere que algunas de los problemas detectados son comunes, y ya informamos que estamos elaborando el estudio de otros países así como de un trabajo que analice las diferencias y carencias no tanto de forma nacional como de forma regional, de tal manera que las soluciones también lo sean, lo que abaratará y posibilitará la consecución de algunos de los objetivos y propuestas que se realizan (CALDERA, 2017).

El análisis que vamos a realizar va a separar las acciones-políticas que debieran orientarse para empresas de aquellas que están orientadas a las instituciones públicas de investigación, especialmente institutos y universidades (aunque en el apartado de universidades, atendiendo a la legislación de cada país, también puede o deben estar integradas las universidades privadas, siempre y cuando los resultados obtenidos por la investigación financiado con dinero público repercuta de alguna manera en la sociedad costarricense, y por supuesto, cualquier tipo de patente no sólo será propiedad de los autores y de su universidad sino también de la entidad financiadora). Es complicado a veces dicha distinción entre empresas y centros públicos de investigación, ya que cada vez es más la fusión y la hibridación de ambas realidades.

\section{$4.1 I+D+i$ para empresas.}

Las acciones orientadas a empresas siempre cuentan con unas características específicas que lo dificultan. La financiación pública no puede ayudar a la empresa privada de 
forma gratuita salvo que sean áreas estratégicas para el país. De tal manera, que siempre se deberán buscar correspondencia para que aquello que repercute en lo privado desde lo público, revierta en cierta manera a la sociedad que sufraga el gasto.

Acción 1. Integración temporal Talento Humano a empresas.

Son pocos los casos en que las instituciones públicas potencian la incorporación del talento humano a empresas. Éstas, por su misión y visión privada, no suelen compartir sus investigaciones ni sus recursos, pero evidentemente toda aquella empresa que quiera incorporar temporalmente a personal altamente cualificado con el fin de asesoría o formación deberá aportar a lo público. No sólo puede realizar la colaboración por medio de factor económicos, sino por la relación de las empresas privadas con las empresas públicas puede ser variadas, tal como la incorporación de becarios procedentes de la universidad, formación gratuita a otras entidades públicas o privadas, etc. En este caso los acuerdos de colaboración se realizarán de forma contractual y con acuerdo entre las partes para que la financiación pública de la incorporación del talento tenga algún tipo de correspondencia. Una vez solucionado esa cuestión, lo positivo de estas incorporaciones siempre temporales será la mejora de la competitividad y de los procesos productivos de la compañía.

Acción 2. Convocatoria competitiva para Proyectos de I+D+i Universidad-Empresa.

También pensando en la obtención de beneficios para lo público, estas convocatorias específicas en las cuáles se busque la relación sinérgica entre empresas, especialmente del tejido productivo y PYMES, e instituciones públicas, especialmente universidades. La relación entre entidades de estos tipos puede generar una ayuda fundamental derivada de la colaboración mutua, de tal manera que las empresas puedan mejorar sus procesos, y las universidades puedan contar con recursos empresarial y con el propio banco de pruebas que es una empresa privada, para el desarrollo de investigaciones conjuntas. Se deberá analizar muy detenidamente las propuestas, siendo conscientes ambas partes que los resultados deberán ser públicos, y en caso de que se generasen patentes sobre dicha investigación éstas serán compartidas por las partes.

Acción 3. Creación de oficina gubernamental para la generación de patentes.

Se entiende procedente esta acción con el fin de mejorar la visibilidad y la seguridad de las investigaciones tanto de PYMES como de instituciones públicas. La creación de una entidad estatal con el fin de potenciar y financiar las tareas orientadas hacia la generación de patentes mejoraría la creación de las mismas, ya que el proceso, a veces complejo, necesita de un asesoramiento profesional por operadores expertos en dicha materia. 


\subsection{I+D+i para universidad y centros de investigación.}

Entendemos que se deben realizar esfuerzos para integrar a la empresa privada, especialmente a las PYMES, en la agenda gubernamental con el fin de que éstos puedan acceder a ayudas y fondos estatales para generar conocimiento y bienes de consumo mejorados para la sociedad. Ahora bien, las empresas tienen un fin claro, y es el ganar dinero por medio de los muchos ingresos con el menor gasto posible. Por lo tanto, esto no hace siempre viable la buena relación con la empresa, a la cual se debe acotar la actuación y asegurar la transición de lo generado con lo público. De ahí, de la existencia de dicha dificultad, y de los fines y misiones que por definición cuenta la empresa privada, es de donde nace nuestro convencimiento que la generación de conocimiento con marcado carácter transversal, que pueda tener validez para la sociedad en sus diferentes vertientes, y que deba hacerse independientemente del rendimiento económico, debe de venir de las instituciones públicas, tanto en la financiación como en la gestión y desarrollo, por ello somos claros defensores de las políticas de inversión y expansión de la investigación orientadas a los centros públicos de investigación, que en la actualidad se definen básicamente como los Institutos de Investigación y las universidades. $Y$ son a estas instituciones a las que se debe dotar de personal y financiación para que sean los motores de la generación de conocimiento y desarrollo social. Una visión de la investigación separado de los ámbitos mercantilistas que le otorgan independencia, flexibilidad y autonomía. Costa Rica cuenta con una estructura estable de Institutos de investigación y Universidades que son, y deben potenciarse, como los principales pilares sobre los que se sustenta y desarrolla la investigación costarricense, y son a estas instituciones, con sus características y sus puntos fuertes y de mejoras, a los que se les debe brindar las herramientas adecuada para la consecución de sus objetivos investigadores, además de los docentes que garantice la transmisión del conocimiento a los jóvenes del país.

Acción 1. Promover las estancias de investigadores extranjeros en centros del país.

Llevada a cabo en otros países de América Latina, especialmente y de manera decidida por Ecuador durante el mandato del Presidente Rafael Correa, la contratación de larga duración de investigadores extranjeros al sistema nacional de investigación es una medida que puede y debe tener grandes resultados para la evolución de los grupos de investigación y la potenciación de líneas de trabajo. Esta acción, de alto coste económico dependiendo del número de investigadores que se quiera incluir, cuenta con varias ventajas: a) sinergias generadas con investigadores extranjeros, b) aumento en la visibilidad internacional, c) aumento en la producción científica de alto nivel, c) aumento en la generación de patentes, d) mejora en la capacitación de los investigadores nacionales, f) formación para investigadores y jóvenes en disciplinas en las que se realiza investigación y desarrollo. Normalmente estos 
investigadores, que se incorporarían al sistema durante al menos un año, no solamente realizarán labores de investigación, sino que en muchos casos transmitirán conocimiento por medio de charlas, cursos, seminarios, talleres, de tal manera que el conocimiento se socializará entre los investigadores y la sociedad en general.

Además de contar con investigadores de primer nivel de otros países también se podrá llevar a cabo la repatriación de investigadores nacionales que estén trabajando fuera de Costa Rica. Suele ser normal que los investigadores de primer nivel no encuentren un lugar para la investigación en sus países de origen, y son unos pocos los países los que están dispuestos a financiar el pago de salarios y de infraestructuras para desarrollar investigación de altísimo nivel. Esta acción por lo tanto podría promover tanto la captación temporal de talento extranjero como la repatriación del talento nacional que emigró al exterior.

Como les decíamos, acción muy potente, con alto coste dependiendo del número de total de investigadores que se quieran incorporar. No obstante, estimamos que los beneficios que acarrean son muchos e importantes, y que deberá ser valorado de forma pormenorizada, tanto en lo referente a la implementación de la política concreta como en la evaluación de perfiles y de candidatos potenciales para ser parte de la acción. La consecución de los objetivos de esta acción estará directamente relacionada con la definición y realización de contratación de perfiles determinados.

Tal y como señalamos, estos investigadores se incluirán en los sistemas Universitarios o de Centros de Investigación, es decir, el gobierno costarricense financiará la incorporación de estos investigadores con el fin de "cederles" a los institutos de investigación y universidades públicas. Además de experiencia y calidad en la producción científica e investigadora, a estos investigadores se les debería solicitar contar con el grado de Doctor (PhD), de tal manera que pudieran igualmente codirigir tesis doctorales y mejorar el sistema educativo de último nivel, el orientado claramente a la investigación y a la docencia.

Acción 2. Formación en maestrías y doctorados en el extranjero.

La forma tradicional y clave para mejorar la formación es normalmente el intercambio. Una vez que el sistema nacional de educación ha otorgado todo su potencial, es bueno dar oportunidad a los jóvenes investigadores para seguir formándose por medio de la realización de estudios y estancias de alto nivel en otras universidades, especialmente si se pudieran hacer convenios de colaboración con el gobierno de Costa Rica y potencias investigadoras. Potenciar el desarrollo de maestrías y doctorados en el extranjero es a priori una buena solución para la mejora del conocimiento científico. Si en la acción anterior (acción 1) importaba talento externo para integrarse temporalmente en el sistema de Costa Rica, esta nueva acción determina el camino contrario: nuestros investigadores, especialmente aquellos 
que están en formación, se marchan a otros centros -también de forma temporal- con el fin de ampliar sus conocimientos, los cuales deberán reintegrase en el sistema costarricense. Se deberá quedar aclarado, por medio de acuerdos contractuales, que los investigadores que obtengan estas ayudas estarán obligados a reintegrases al sistema por al menos el mismo tiempo que se haya ayudado y financiado al desarrollo investigador en el extranjero.

Becar a los mejores jóvenes investigadores para que sigan formándose, pero con la contraparte de estar obligados a volver e incorporar al Sistema costarricense, de lo contrario se deberá devolver todo lo invertido por parte del becado. Por lo tanto, debe ser garantistas en que la inversión debe revertir en el país y por lo tanto en la sociedad, por medio del desarrollo de proyectos para Costa Rica.

Pero además de becas predoctorales, debe entenderse como becas de carácter formativo a todo investigador del Sistema Nacional. Todo aquel que quiera mejorar en su formación tendrá cabida en esta acción. Por lo tanto, igualmente se podrá otorgar financiación de becas postdoctorales, así como a investigadores de alto nivel que quieran mejorar su formación por medio de la relación investigadoras con otros organismos internacionales.

Acción 3. Creación de Institutos de Investigación de carácter público.

Aunque Costa Rica cuenta con Institutos de Investigación, seguramente se debería potenciar su desarrollo, así como la creación de nuevos institutos. Es importante, sino vital, la generación de institutos no sólo de carácter científico-técnico, sino que parece relevante generar institutos en los ámbitos de las Ciencias Sociales, que al final son desde el estudio y análisis de estas temáticas desde donde existe un mayor desarrollo social para la sociedad costarricense. Entendemos además que debería existir una institución gubernamental propia que agrupe diferentes institutos e instituciones de investigación, un Consejo Superior de Investigación que controlara y potenciara la investigación en el país, y que colaborase y fuese de la mano con las universidades ya existentes en el país.

Acción 4. Creación de spin-off de base tecnológica.

Debe potenciarse la creación de empresas con base tecnológica en el marco de las instituciones públicas de investigación. Empresas que desarrollen y se relacionen con el ámbito empresarial, aunque siempre con una base y una filosofía de carácter social. Sería el puente entre la empresa privada y los desarrollos tecnológicos, lo que conllevaría una generación de conocimientos y también de riqueza y empleo.

Acción 5. Implementación y mejora de los canales de distribución de la Ciencia costarricense

Es oportuno la generación de nuevas formas de difusión de la información científica generada por el sistema, no parece oportuno que todo aquello que se ha generado gracias al esfuerzo y financiación del Sistema Nacional de investigación no sea conocido por la sociedad 
científica especialmente y tenga su correspondencia y visibilidad social. Revistas científicas, congresos y publicaciones monográficas siguen siendo las fórmulas, aunque también deberemos potenciar los repositorios públicos que garanticen el acceso a los contenidos (DORA, 2012).

Potenciar el desarrollo de congresos por medio de convocatorias competitivas entre universidades e institutos públicos, que garantice la existencia periódica de eventos de carácter internacional que garantice la presencia y difusión de los contenidos generados en nuestro sistema y también conocer los desarrollos de otros países.

Sobre las revistas científicas, se debe potenciar y asesorar desde el Sistema la generación de nuevas revistas y la mejora de las ya existentes para mejorar su visibilidad y su calidad, estando presente en los índices de calidad, además de generar índices nacionales propios que ponderen la calidad y, la financiación si fuera oportuno, de las revistas costarricense.

Acción 6. Creación de Repositorio institucional público como método de difusión y evaluación.

Entendeos que la difusión de la ciencia generado con dinero público es una obligación del país. Ya sea generado por proyectos públicos o por personal que sus salarios proceden de la financiación del estado (como los trabajadores de la universidad), la investigación debe ser difundida por canales abiertos, ya que los derechos de autor son compartidos con la institución que paga los salarios. Si partimos de esta premisa, cualquier labor de investigación debe traducirse en una difusión de los resultados, y éstos deben ser garantizados para que sean accesibles por la comunidad investigadora y por la sociedad.

Para ello, una opción, es generar repositorios públicos en los cuáles se faciliten los contenidos independientemente de que estén publicados o no en otras revistas o fuentes de información. Además, se debe potenciar la publicación siempre en acceso abierto. Pero si esto no se consigue, los investigadores estarán obligados a difundir los contenidos en este formato abierto, que son los repositorios.

Igualmente, estas herramientas pueden ayudar a una nueva fórmula de evaluación de la Ciencia, ya que, para incorporarse a este repositorio institucional, se deberá pasar controles de calidad que identifiquen el nivel y valía de los trabajos expuestos en el repositorio público. Acción 7. Registro de grupos de investigación

Asociado a la creación de un repositorio nacional, y seguramente desde la misma institución, se podría generar un registro de grupos de investigación con el fin de determinar quién está desarrollando labores de investigación y a qué se está dedicando. Conocer los grupos mejorará las sinergias y los contactos entre los mismos, tanto a nivel nacional como internacional, ya que además se deberán indicar cuáles son las principales líneas del equipo y 
trabajos actuales. Igualmente, estos grupos de investigación tendrían una financiación mínima que garantizase la capacidad de funcionamiento partiendo de necesidades instrumentales y materiales.

Acción 8. Agencia de evaluación de la Ciencia en Costa Rica

Relacionado con la creación de un repositorio, y en cierta manera relacionado con la generación de un registro de grupos de investigación, se debería crear una agencia de evaluación en Costa Rica que analice tanto al personal investigador (para categorizarlo e incentivarlo) así como la producción científica de grupos y de investigadores del Sistema Nacional de Ciencia y Tecnología.

Además, serviría para analizar toda la red incluido las universidades, los centros que la integran y las titulaciones que la conforman.

Sin duda, puede ser un organismo complejo en su planificación y aún más complicado su implementación, pero sería la institución que otorgaría validez, calidad y viabilidad a todo el sistema público de investigación y educación superior.

\section{Conclusiones}

A la vista de todo lo expuesto anteriormente, es complicado concluir cuáles son las mejores pautas para una política de actuación en materia de investigación y desarrollo, habida cuenta de que además de los intereses políticos y estratégico del país, se ha de tener muy en cuenta las posibilidades presupuestarias con las que cuenta Costa Rica para mejorar su sistema de investigación.

No obstante, sí que existen indicios para determinar que aún queda mucho camino por recorrer. El análisis de datos no deja en mala posición la publicación costarricense, siempre y cuando la comparemos con los países de la región, mucho peor son las comparativas si son realizadas con todo América Latina o si la posicionamos en su lugar en el mundo, en el ámbito de la investigación. No obstante, no parecen números bajos atendiendo a la población total del país y al número total de investigadores existentes. Número, el de investigadores, que se encuentra en la media mundial pero que está lejos de los países desarrollados y que conforman la OCDE, lugar que estimamos debería intentar alcanzar un país pujante como Costa Rica.

Aunque volvemos a remarcar que es líder de la región, esto no deja de ser el compararse con países con escasa pujanza investigadora, y los cuáles adolecen de una inversión potente en materia de investigación y desarrollo (vamos a exceptuar a Panamá que cuenta al igual que en Costa Rica con procesos y procedimientos para la gestión de la investigación en el país). Pero el dato más revelador de todos es la inversión en Investigación y 
Desarrollo puesto en relación con el Producto Interior Bruto del país. Costa Rica debe realizar más y mejores esfuerzos para financiación y potenciar su investigación, de una financiación más alta podremos obtener mejores resultados. La relación entre inversión y resultados es incuestionable en materia de investigación.

Además de la financiación, que volvemos a señalar es un elemento clave, dichos recursos deben gestionarse de manera oportuna y eficaz. Uno de los puntos fuertes del Sistema de Ciencia en Costa Rica es que se cuenta con una institución importante, con experiencia y trayectoria, en la gestión de financiación y desarrollo de programas en materia de investigación. Por lo tanto, se cuenta con las estructuras estables para canalizar todo el proceso investigador de carácter competitivo y finalista.

Debe mejorarse no solo la investigación sino la difusión. Debe garantizarse por medio de algunas de las herramientas expuestas, que lo generado a partir de la investigación financiada con dinero público esté al alcance de todos los costarricenses, y que no se difundan en publicaciones privadas y de pago, algunas de las cuáles son necesarias importantes desembolsos para acceder a los contenidos, mientras que otras cuentan con largos periodos de embargo para poder acceder a los contenidos. Apostamos claramente por la creación de repositorios públicos y revistas en acceso abierto para la difusión de la Ciencia no sólo en Costa Rica sino en todos los países del Mundo.

Sobre políticas concretas que pudieran implementarse, incidir que hemos propuesto un catálogo a toda luz imposible de llevar a cabo con la financiación actual que el Gobierno de Costa Rica dedica a la Investigación y al Desarrollo, habrá de ponderarse la validez y la valía de algunas de las propuestas para determinar cuál o cuáles pueden ser incluidas y cómo llevarlas a cabo, teniendo muy presente el esfuerzo de financiación necesario para lograr tales fines.

Entendemos que la Ciencia en Costa Rica tiene mucho margen de mejora y no solo mejorando la financiación esta vía es posible. Existen también decisiones políticas que marcan el alineamiento y posicionamiento investigador (en este caso los ejes estratégicos del país), y cómo relacionarse con los procesos productivos y las empresas privadas.

Es necesario recuperar las Ciencias Sociales como elemento vertebrador de un sentir colectivo. El descuido en las Ciencias Sociales, Humanas y en las Artes debe ser rápidamente contrarrestado con nuevos programas que hagan que la sociedad se sienta más cercana del proceso creativo y de investigación, además de que se vean reflejados claramente como objetos pacientes de todo lo generado y como objetivos de la Ciencia. No sólo acercar la Ciencias a la sociedad costarricense, sino que se sientan implicados en la misma.

Costa Rica es un país en crecimiento, en cierta manera en formación y transformación constante, donde la mejora es el camino natural, donde sus condiciones medioambientales y 
posición geográfica la hacen estratégicamente fundamental e imprescindible en la Región. Desarrollemos esta potencialidad por medio de la generación de nuevas directivas y directrices para generar políticas de inversión de lo púbico y de lo privado, abriendo la posibilidad a la colaboración internacional (colaboración no es cesión) y a la implementación de nuevas políticas de inversión en materia de investigación.

Potenciemos y desarrollos, por medio de políticas públicas de Ciencia, la PURA VIDA costarricense.

\section{Referencias}

BANCO MUNDIAL (2018). https://www.bancomundial.org/ Consultado en: 7/10/2018.

CALDERA SERRANO, Jorge. Análisis de las políticas de I+D+i en Panamá implementadas por SENACYT. Revista Ibero-Americana de Ciência da Informação, v. 12, n. 3, p. 956-975, 2019. https://doi.org/10.26512/rici.v12.n3.2019.24441

CALDERA SERRANO, Jorge. Desarrollo de un Instituto supranacional para la salvaguarda de los archivos y colecciones audiovisuales para América Latina y el Caribe. Cuadernos de Documentación Multimedia, España, v. 28, n. 2, p. 136-146, 2017. ISSN: 1575-9733

COSTA RICA. CONSEJO NACIONAL PARA INVESTIGACIONES CIENTÍFICAS Y TECNOLÓGICA. Plan operativo institucional (POI): 2018.

COSTA RICA. CONSEJO NACIONAL PARA INVESTIGACIONES CIENTÍFICAS Y TECNOLÓGICAS (2018 b). www.conicit.go.cr/ Consultado:07/10/2018.

COSTA RICA. Ministerio de Ciencia, Tecnología y Telecomunicaciones (2018). https://micit.go.cr/. Consultado: 7/10/2018.

DORA. Declaración de San Francisco de evaluación de la Ciencia: poniendo ciencia en la evaluación de la investigación. 2012.http://blogs.ujaen.es/cienciabuja/wpcontent/uploads/2013/10/dora.pdf Consultado en: 7/10/2018.

HAPPY PLANET INDEX (2018). http://happyplanetindex.org/ Consultado en: 7/10/2018.

INDEXMUNDI (2018). https://www.indexmundi.com/es/ Consultado en: 7/10/2018.

PIEDRA SALOMÓN, Yelina; Martínez Rodríguez, Ailín. Producción científica. Ciencias de la Información, v. 39, n. 3, p. 33-38, 2007.

SCIMAGO (2018). https://www.scimagojr.com/ Consultado en:07/10/2018.

UNESCO (2018). https://es.unesco.org/ Consultado en: 7/10/2018.

WIKIPEDIA. Costa Rica. https://en.wikipedia.org/wiki/Costa Rica Consultado en: 7/10/2018. 